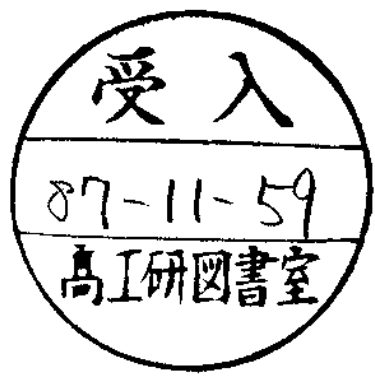

CERN-TH. 4824/87

$\mathrm{MAD} / \mathrm{TH} / 87-23$

\title{
UNIVERSALITY OF THE MASS SPECTRUM IN CLOSED STRING MODELS
}

\author{
I. Antoniadis ${ }^{*}$ ) and John E11is \\ CERN - Geneva
}

and

D.v. Nanopoulos

Physics Department, University of Wisconsin Madison, WI 53706, USA

\section{A B S T R A C T}

We show that the asymptotic level densities of particle states in closed string theories are universal. They can be written in the form $\mathrm{n}(\mathrm{m}) \sim \mathrm{m}^{-\mathrm{a}} \exp \mathrm{bm}$ where $\mathrm{b}$ depends only on the central charge of the virasoro algebra and is $b=2 \sqrt{ } 2 \pi \sqrt{ } \alpha^{\prime}$ for all type II superstrings and $b=(2+\sqrt{ } 2)$ $\pi \sqrt{ } \alpha^{\prime}$ for all heterotic strings, independently of the number $D$ of space-time dimensions in which they are formulated, whilst $a=D$ has the same dependence on the dimensionality of space-time for both type II and heterotic strings. We comment on the relevance of these results for the classification of string models and for the thermodynamics of hot strings. We speculate that the dependence of $a$ on $D$ may explain why we live in four dimensions.

*) On leave from the Ecole Polytechnique, F-91128 Palaiseau, France. 
Although closed string theory is the only serious candidate for a Theory of Everything including gravity [1], we do not yet know which string model Nature has chosen. Different closed string models [2-8] may be characterized as unitary realizations of the left- and right-moving virasoro algebras with central charges $c=26$ or $c=15$ if there is a world sheet supersymmetry, which are constrained by multiloop modular invariance. The unitary representations of the virasoro algebra with $c<1$ are catalogued [9], but those with $c>1$ are incompletely known and apparently very numerous. It is a matter of opinion whether all these realizations of the Virasoro algebra should be regarded as different ground states of the same string theory, or as intrinsically different theories. However, it is clear that the low-energy spectra of all these string models are very varied [10,11]. Indeed, today's central phenomenological problem is that of finding the string model (unitary realization of the Virasoro algebra) which describes the spectrum of 1 ight particles and their interactions.

If there is any universality of the spectra in string models, it can only appear at high masses, not at low masses. It has been known [12] since the early days of dual models for hadrons that the asymptotic expansion for the level density in a string model starts with a term [12-17]

$$
n(m) \sim c m^{-a} e^{b m}
$$

for 1 arge masses $m$, where $b=0\left(\sqrt{ } \alpha^{\prime}\right)$, a is a number and $c=0\left(\sqrt{ } \alpha^{\prime}\right)^{a}$. An asymptotic level density of the form (1) had been proposed previously for hadrons by Hagedorn [13], on the basis of a statistica] bootstrap model. He went on to argue that a level density such as (1) leads in general to a phase transition at a temperature $\mathrm{T}_{\mathrm{H}}=1 / \mathrm{b}$. Which thermodynamic quantities become now divergent at the Hagedorn temperature depends on the exponent in the prefactor of Eq. (1). Nowadays we believe that the quarks inside hadrons become deconfined at temperatures $\mathrm{T}>\mathrm{T}_{\mathrm{c}}=0\left(\mathrm{~T}_{\mathrm{H}}\right)$, and that the new phase is a strongly (weakly) coupled quark-gluon gas at temperatures just ( $f a r$ ) above the critical temperature $\mathrm{T}_{c}$. There have naturally been suggestions that since the mass spectra in string models also behave like (1), there will be a phase transition at some new Hagedorn temperature $\mathrm{T}_{\mathrm{H}} \sim 1 / \sqrt{ } \alpha^{\prime} \sim 10^{19} \mathrm{GeV}$, but the nature of the high-temperature phase is not yet clear [15-18].

In this paper we calculate the parameters $b$ and $a$ characterizing the asymptotic level density of all the closed string theories of phenomenological interest, formulated in any number $D$ of space-time dimensions. We find remarkable universality properties. The parameter $b$ depends only on the central charge of the Virasoro algebra. All type II superstring models, which have $c=15$ for both leftand right-movers, have $\mathrm{b}=2 \sqrt{ } 2 \pi \sqrt{ } \alpha^{\prime}$. All heterotic string models, which have $c=15$ for left movers and $c=26$ for right movers, have $b=(2+\sqrt{ } 2) \pi \sqrt{ } \alpha^{\prime}$. These results are independent of $\mathrm{D}$. However, the exponents in the prefactors always have the same 
dimension dependence: $a=D$ independently of the string type ${ }^{*}$. The differences between the parameters $b$ for type II and heterotic strings suggest that they are intrinsically different theories, and that one cannot, for example, identify the $\mathrm{D}=10$ heterotic string as some asymmetric ground state of the $\mathrm{D}=26$ bosonic string [20]. However, al.1 type II (a11 heterotic) string models could still be variants of the same (another) theory. We conclude by commenting on the interpretation of (1) as implying a phase transition at some temperature $\mathrm{T}_{\mathrm{c}} \sim \mathrm{T}_{\mathrm{H}}=\mathrm{l} / \mathrm{b}$, arguing that the nature of the phase transition and of the new phase can only be understood in the context of what we would nowadays regard as a nonperturbative formulation of string theory. We also speculate that the dependence of a on $D$ may explain why we 1 ive in four dimensions.

We now proceed to the calculation of the asymptotic level density of particle states following the method of Ref. [16]. It can easily be derived from the zerotemperature string "partition function" $Z$ which is related to the one-loop vacuumto-vacuum amplitude $v$ :

$$
V=\int_{M} \frac{d \tau d \bar{\tau}}{(\operatorname{Im} \tau)^{2}} Z_{0} Z(x)
$$

In Eq. (1), $z_{0}$ is the contribution of the bosonic zero-modes, $x=e^{2 i \pi \tau}$ and the integration over the complex parameter $\tau$ of the torus is performed inside the fundamenta]. region $M$ [18]. The degeneracy of states $P_{L(R)}(N)$ corresponding to the Nth Jevel of the left- (right-) moving sector of the closed string can be found by expanding $Z(x)$ in powers of $x$ and $\bar{x}$

$$
Z(x)=\sum_{N, \bar{N}=0}^{\infty} P_{L}(N) P_{R}(\bar{N}) x^{N} \bar{x}^{\bar{N}}
$$

The physical states of the closed string with mass $m=2\left(\sqrt{ } / \sqrt{ } \alpha^{\prime}\right)$, where $\alpha^{\prime}$ is the Regge slope, satisfy the condition $N=\bar{N}$ and their multiplicity is then $P_{L}(N) P_{R}(N)$. Thus, the density of states $n(m)$ of mass $m$ is given by

$$
n(m)=\frac{\alpha^{\prime}}{2} m P_{L}(N) P_{R}(N) ; N=\frac{\alpha^{\prime}}{4} m^{2}
$$

By inspection of Eqs. (3) and (4), we conclude that the asymptotic behaviour of $n(m)$ for large $m$ is related to the $x \rightarrow 1^{-}$behaviour of the "partition function" $z$. We will show that this behaviour depends only on genera] properties of the theory such as of the existence or not of world-sheet supersymmetry and the space-time dimensionality $D$, and it does not depend on the particular features of the model. We will use the fermionic formulation of string theories in $D \leqslant 10[6-8]$ and for pedagogical reasons we will restrict ourselves to the class of models obtained when

*) After these results were obtained, but before they were written up, we received an advance copy of Ref. [19] in which many of the same results are reported. A] though our results are identical where they overlap, the different nuances of our interpretation encouraged us to write the present paper. 
the extra two-dimensional fermions are only periodic or antiperiodic around the two non-contractible loops of the torus. The generalization to fermions with arbitrary boundary conditions [8] is straightforward and does not alter our results. We will discuss later their extension to models containing components with $\frac{1}{2}<c<1$ which do not seem to have a simple description in terms of free fermions.

The world-sheet field content of a D-dimensional fermionic string has in addition to the $D$ bosonic co-ordinates $2(26-D)$ additional two-dimensional fermions when there is no world-sheet supersymmetry, otherwise there are in addition to the $D$ bosonic co-ordinates and their fermionic superpartners 3(10-D) world-sheet fermions among which supersymmetry is non-linearly realized [4]. Following the notation of Ref. [7], $z$ is given, for a generic fermionic string model of this type, by

$$
Z=Z_{B}^{D-2} \sum_{\alpha, \beta \in \equiv} c(\alpha / \beta)[\alpha \mid \beta]
$$

where the first factor is the contribution of the D-2 transverse bosonic co-ordinates and $[\alpha / \beta]$ denotes the contribution of the sector where the fermions of the set $\alpha$ are periodic in the $\sigma$-direction and those of $\beta$ are periodic in the $\tau$-direction of the torus: the $c(\alpha / \beta)$ are coefficients which are equal to \pm 1 for every pair of sets $\alpha, \beta$ in $\Xi$, according to the rules of Ref. [7] which are a consequence of multiloop modular invariance. The $[\alpha / \beta]$ can be expressed in terms of the Jacobi theta functions $\theta_{i}(x)$ and of the Dedekind-eta function $\eta(x):[21]$ :

$$
\begin{aligned}
{[\alpha \mid \beta] } & =\left(\frac{\theta_{1}}{\eta}\right)^{n_{L}(\alpha n \beta)}\left(\frac{\bar{\theta}_{L}}{\bar{\eta}}\right)^{n_{R}(\alpha n \beta)}\left(\frac{\theta_{2}}{\eta}\right)^{n_{L}(\alpha-\alpha n \beta)}\left(\frac{\vec{\theta}_{2}}{\bar{\eta}}\right)^{n_{R}(\alpha-\alpha n \beta)} \cdot \\
& =\left(\frac{\theta_{4}}{\eta}\right)^{n_{L}(\beta-\alpha n \beta)}\left(\frac{\bar{\theta}_{4}}{\bar{\eta}}\right)^{n_{R}(\beta-\alpha n \beta)}\left(\frac{\theta_{3}}{\eta}\right)^{n_{L}(F-\alpha \cup \beta)}\left(\frac{\vec{\theta}_{3}}{\bar{\eta}}\right)^{n_{R}(F-\alpha \cup \beta)}
\end{aligned}
$$

where $n_{L(R)}(A)$ denotes one half the number of left- (right-) moving fermions of $A$, and $F$ is the set of all fermions. Finally, $Z_{B}$ is given by

$$
z_{8}=\frac{1}{\eta \bar{\eta}}
$$

We note that $\theta_{1}$ vanishes identically, while when $x \rightarrow 1, \theta_{4} / \eta$ vanishes and $\theta_{2} / \eta \approx \theta_{3} / \eta$. Thus, $z$ becomes

$$
Z \underset{x \rightarrow 1}{\sim} \frac{z}{(\eta \bar{\eta})^{D-2}} \sum_{\alpha \in \equiv} c(\alpha \mid \phi)\left(\frac{\theta_{2}}{\eta}\right)^{n_{L}(F)}\left(\frac{\bar{\theta}_{2}}{\bar{\eta}}\right)^{n_{R}(F)}
$$

But $c(\alpha / \phi)=\delta_{\alpha}=+1$ or -1 according to whether the states are space-time bosons or fermions. For our purpose, both contributions are additive and we find 


$$
Z \underset{x \rightarrow 1}{\sim} \frac{2}{(\eta \bar{\eta})}\left(\frac{\theta_{z}}{\eta}\right)^{\eta_{L}(F)}\left(\frac{\bar{\theta}_{2}}{\bar{\eta}}\right)^{n_{R}(F)} .
$$

We finally need to evaluate the $x \rightarrow 1$ behaviour of $\eta$ and $\theta_{2} / \eta$. This can be achieved by defining $\tau \equiv-1 / \tau^{\prime}$ or equivalently $x^{\prime} \equiv e^{4 \pi^{2} / \ell n x}$ which goes to zero as $x \rightarrow 1^{-}$, and using the modular transformations of the corresponding functions. Indeed, we find

$$
\eta^{-1}(x)=\left(-\frac{1}{2 \pi} \ln x\right)^{1 / 2} \eta^{-1}\left(x^{4}\right) \underset{x \rightarrow 1^{-}}{\rightarrow}\left(-\frac{1}{2 \pi} \ln x\right)^{1 / 2} e^{-\frac{\pi^{2}}{6 \ln x}}
$$

and

$$
\frac{\theta_{2}}{\eta}(x)=\frac{\theta_{4}}{\eta}\left(x^{\prime}\right) \underset{x \rightarrow 1}{\longrightarrow} e^{-\frac{n^{2}}{6 \ln x}}
$$

The behaviour of the degeneracy of states $P_{L(R)}^{a s}(N)$ for large $N$ is given by a contour integral, e.g.,

$$
P_{L}^{a s}(N)=\frac{1}{2 \pi i} \int_{C} \frac{d x}{x^{N+1}} Z_{L}^{\text {as }}(x)
$$

which can be evaluated by a saddle-point approximation. In fact, $\mathrm{z}^{\text {as }}$ has the form

$$
z^{\text {as }} \sim c(\ln x)^{A / 2} e^{-B \frac{n^{2}}{6 \ln x}}
$$

and therefore

$$
P(N) \sim C \int(d \ln x)(\ln x)^{A / 2} e^{-N \ln x-B \frac{\pi^{2}}{6 \ln x}}
$$

which, by change of variables $\ln x=1 / \sqrt{ } \mathrm{N}$ y becomes

$$
\begin{aligned}
& P(N) \sim C N^{-\frac{A}{4}-\frac{1}{2}} \int d y y^{A / 2} e^{-\sqrt{N}\left(y+\frac{n^{2}}{6} \frac{B}{y}\right)} \\
& =2 \sqrt{n}\left(\pi \sqrt{\frac{B}{6}}\right)^{A / 6}\left[\sqrt{\frac{8}{3}} \pi \sqrt{B}\left(\pi \sqrt{\frac{B}{6}}\right)^{3 / 2}\right]^{-1 / 2} c N^{-\frac{3}{4}-\frac{A}{4}} e^{2 \sqrt{\frac{B}{6}} \pi \sqrt{N}}
\end{aligned}
$$

where in the last step we applied the saddle-point approximation. By substitution of ( $12 b$ ) into (4) we find 


$$
n(m) \sim m^{-a} e^{b m}
$$

with

$$
a=2+\frac{1}{2}\left(A_{L}+A_{R}\right) \text { and } b=\frac{\sqrt{B_{1}}+\sqrt{B_{R}}}{\sqrt{6}} \pi \sqrt{\alpha^{\prime}} \text {. }
$$

From Eqs. (8b), (9) and (11), we have $A_{L}=A_{R}=D-2$ which gives $a=D$ and $B=12$ or 24 according to whether the corresponding sector is world-sheet supersymmetric or not. Therefore $b=(2+\sqrt{ } 2) \pi \sqrt{ } \alpha^{\prime}$ for the heterotic, $2 \sqrt{ } 2 \pi \sqrt{ } \alpha^{\prime}$ for the type II and 4 for the closed bosonic string.

It is clear that the coefficients $B_{L}$ and $B_{R}$ in (13) are just the fermionic contributions to the central charges of the left- and right-moving virasoro algebras. As was commented earlier, there are unitary representations [9] of the Virasoro algebra with $\frac{1}{2}<c<1$ that do not have a simple free-field interpretation. These could also be included as building blocks in constructing string models with a total $c=26(15)$ for sectors without (with) world-sheet supersymmetry. The results (11) to (13) can be generalized trivially to include such possibilities, since the relevant behaviours of the partition functions for $c<1$ models are known [22]

$$
z^{\text {as }}(c){\underset{x \rightarrow 1^{-}}{ }}^{-\frac{c 7^{2}}{6 \ln x}}
$$

which would multiply (11) and hence add a contribution $c$ to $B$. Thus the result for $\mathrm{b}$ in Eq. (13) can be written as

$$
b=\frac{\sqrt{c-2}}{\sqrt{6}} \pi \sqrt{\alpha^{\prime}}
$$

for a non-supersymetric sector, and

$$
b=\frac{\sqrt{c-3}}{\sqrt{6}} n \sqrt{\alpha}
$$

for a supersymmetric sector.

One could proceed to calculate the one-loop finite-temperature partition function using a canonical ensemble, finding a Hagedorn temperature $T_{H}=1 / b$. If the partition function were to diverge as $\mathrm{T} \rightarrow \mathrm{T}_{\mathrm{H}}-\varepsilon$, then $\mathrm{T}_{H}$ could be interpreted as a maximum temperature. In fact, it is well known [14] that if 


$$
a>\frac{D+3}{2}
$$

then the pressure $p$ and the density $\rho$ go to constants as $T \rightarrow T_{H}-\varepsilon$, and there is the possibility of a phase transition at a critical temperature $T_{c}=T_{H}$. Our result $a=D$ implies that $p$ and $\rho$ do indeed go to constants at $T_{H}$ for all dimensions $\mathrm{D}>4$, which includes all the models of interest. We will speculate later what happens when $\mathrm{T}>\mathrm{T}_{\mathrm{H}}$.

We conclude by discussing the interpretation of our results. The universality of the exponential growth rates b for different type II (heterotic) strings, whatever the number of space-time dimensions in which they are formulated is consistent with the view that they are all different variants of the same (another) string theory. This universality is also consistent with the view that there is no intrinsic difference between external space-time and internal degrees of freedom. There is no need to interpret the internal degrees of freedom as any sort of compactified space-time. The fact that the exponential growth rates b are different for sectors with and without world-sheet supersymmetry goes against the view [20] that these can be regarded as different variants of the same theory. In particular, the difference in the growth rates for the ten-dimensional heterotic string and for the 26-dimensional bosonic string suggests that the former is intrinsically different from the latter, and cannot be regarded as some asymmetric ground state of $i t^{*}$ )

Although our results are suggestive of a phase transition at a temperature $T=T_{C}=0\left(T_{H}=1 / b\right)$, they are not conclusive. Higher orders of the string topological expansion, even infinite resummation or non-perturbative effects could play an essential rôle. There is no reason to expect that string scattering at $s \sim \mathrm{T}_{\mathrm{H}}^{2}$ is so weak as to be negligible, so that treating hot string as an ideal gas at temperatures $T \rightarrow T_{H}-\varepsilon$ is unlikely to be valid. This is certainly true at larger values of $s$. It is known [24] that an accurate treatment of the Regge 1 imit: ( $s \rightarrow \infty$, $t$ fixed) in string theory requires, at the very least, resumation of an infinite number of terms in the string topological expansion. This problem is worse for contemporary string theory than it was for hadrons, since the leading Regge trajectory, that of the graviton, has $J=2$ at $t=0$. This resumation could conceivably renormalize the Regge trajectories and alter the asymptotic spectrum in an essential way. High-energy, fixed-angle string-string scattering also has some unusual features [25]. The asymptotic behaviour of the genus $G$ term in the topological expansion is $\sigma \sim \exp (-s \ell n s / G+1)$. To any finite order in perturbation theory, this gives an interaction rate $\Gamma(V s) \equiv n(/ s) \exp (-\operatorname{sens} / G+1) \rightarrow 0$ as $/ s \rightarrow \infty$,

*) See also Ref. [23] for an independent argument in this direction. 
which is much less than $H(/ s) \equiv\left(/ s n(/ s) / m_{p}^{2}\right)^{\frac{1}{2}}$ and hence not sufficient by itself to keep high energy string states in thermal equilibrium if one uses naive Big Bang cosmology as would have been valid for hadrons. However, the fact that higherorders of the string topological expansion fall off even slower ( exp-slns/G+1) at large $s$, indicates that an infinite resumation of all terms in the loop expansion is necessary, and very 1ikely non-perturbative effects also. These might restore the justification for treating the early universe using thermodynamics for hot strings. However, in this case the properties of a hot string gas would only be calculable using techniques which go beyond any perturbative formulation of string theory, just as a hot hadronic gas is understood in terms of quarks and gluons which are not obtainable perturbatively from hadrons.

If we assume that the Universe was at one time in another such phase, this could explain why we now live in four dimensions. As we have seen above; string models in less than four dimensions would have $P, \rho \rightarrow \infty$ as $T \rightarrow T_{H}-\varepsilon$, and so could not be obtained from a phase with finite values of $p, p$. The dependence of a on the number of space-time dimensions suggests that the entropy is extremized for $D=4$. We do not yet have a complete argument how this extremal choice is selected at the phase transition, but we do note that the specific heat is infinite if $D=4$.

It is amusing to speculate that this analogy with the QCD phase transition can be carried further. In the case of the hadron-quark transition, the string tension is believed to go to zero as $T \rightarrow T_{c}-\varepsilon$, allowing confined quarks and gluons to become more and more separated and eventually deconfined. It is natural to conjecture that something similar may occur to hot string states, with the string tension going to zero as $T \rightarrow T_{c}=0\left(T_{H}\right)-\varepsilon$, so that individual "particle" states, gravitons included, become in some sense larger and larger until... 


\section{REFERENCES}

[1] See, e.g.: M.B. Green, J.H. Schwarz and E. Witten - Superstring Theory (Cambridge University Press, 1987) and references therein.

[2] D.J. Gross, J.A. Harvey, E. Martinec and R. Rohm - Phys.Rev.Lett. 54 (1985) 502; Nucl.Phys. B256 (1985) 253; Nuc1.Phys. B257 (1986) 75.

[3] L. Dixon, J.A. Harvey, C. Vafa and E. Witten - Nucl.Phys. B261 (1985) 651; Nucl.Phys. B274 (1986) 286.

[4] I. Antoniadis, C. Bachas, C. Kounnas and P. Windey - Phys.Lett. 171B (1986) 51 .

[5] K.S. Narain - Phys.Lett. 169B (1986) 41;

W. Lerche, D. Lust and A.N. Schellekens - Nucl.Phys. B287 (1987) 477.

[6] H. Kawai, D.C. Lewellen and S.H.H. Tye - Phys.Rev.Lett. 57 (1986) 1832; Nuc I.Phys. B288 (1987) 1.

17] I. Antoniadis, C. Bachas and C. Kounnas - Nucl.Phys. B289 (1987) 87.

[8] I. Antoniadis and C. Bachas - CERN Preprint TH. 4767 (1987).

[9] A.A. Belavin, A.N. Polyakov and A.B. Zamolodchikov - Nuc1.Phys. B241 (1984) 333 ;

D. Friedan, Z. Qiu and S. Shenker - Phys.Rev.Lett. 52 (1984) 1575.

[10] J. Ellis - Lectures at the Cargese Summer School (1987) and references therein.

[11] I. Antoniadis, J. Ellis, E. Floratos, D.V. Nanopoulos and T. Tomaras Phys.Let t. 191B (1987) 96;

S. Ferrara, L. Girardello, C. Kounnas and M. Porrati - Phys.Lett. 191B (1987) 358; Phys.LEt.t. 194B (1987) 358.

[12] S. Fubini and G. Veneziano - Nuovo Cimento 64A (1969)811;

K. Bardakci and S. Mandelstam - Phys.Rev. 184 (1969) 1640;

S. Fubini, D. Gordon and G. Veneziano - Phys.Lett. 29B (1969) 679.

[13] R. Hagedorn - Nuovo Cimento Supp1. 3 (1965) 147; Nuovo Cimento Suppl. 6 (1968) 311; Nuovo Cimento 52A (1967) 1336; Nuovo Cimento 56A (1968) 1027.

[14] K. Huang and S. Weinberg - Phys.Rev.Lett. 25 (1970) 895.

[15] E. Al.varez - Phys.Rev. D31 (1985) 418; Nuc1.Phys. B269 (1986) 596.

[16] M.J. Bowick and L.C.R. Wijewardhana - Phys.Rev.Lett. 54 (1985) 2485.

[17] S.H. Tye - Phys.Lett. 158B (1985) 388.

[18] J. Polchinski - Commun. Math.Phys. 104 (1986) 37.

[19] M. Axenides, S. EJ.1is and C. Kounnas - Univ, of Washington Preprint, in preparation (1987).

[20] P. Freund - Phys.Lett. 151B (1985) 387;

A. Casher, F. Englert, H. Nicolai and A. Taormina - Phys.Lett. 162B (1985) 121 ;

D. Lïst - M.P.I. Preprint MPI-PAE/PTh 3/87 (1987);

F. Englert, H. Nicolai and A. Schellekens - Nucl.Phys. B274 (1986) 315. 
[21] See, e.g.: D. Mumford - Tata Lectures on Theta I and II, Birkhäuser (1983 and 1984 ).

[22] J.L. Cardy - Nucl.Phys. B270 [FS16] (1986) 186;

C. Itzykson and J.B. Zuber - Nuc1.Phys. B275 [FS17] (1986) 580;

A. Capel1i, C. Itzykson and J.B. Zuber - Nuc1.Phys. B280 [FS18] (1987) 445.

[23] P. Oxland - Nuc1.Phys. B278 (1986) 790.

[24[ D. Amati, M. Ciafaloni and G. Veneziano - CERN Preprint TH. 4782 (1987).

[25] D.J. Gross and P.F. Mende - Princeton University Preprint PUPT-1062 (1987). 